\title{
Effect of Brownian Motion and Thermophoresis on a Nonlinearly Stretching Permeable Sheet in a Nanofluid
}

\author{
A. Falana', 0. A. Ojewale1, T. B. Adeboje ${ }^{2}$ \\ ${ }^{1}$ Department of Mechanical Engineering, University of Ibadan, Ibadan, Nigeria \\ ${ }^{2}$ Department of Mechanical Engineering, The Polytechnic, Ibadan, Ibadan, Nigeria \\ Email: falanaayode@gmail.com, falana.ayo@ui.edu.ng
}

Received 17 December 2015; accepted 21 February 2016; published 24 February 2016

Copyright (C) 2016 by authors and Scientific Research Publishing Inc.

This work is licensed under the Creative Commons Attribution International License (CC BY). http://creativecommons.org/licenses/by/4.0/

c) (i) Open Access

\begin{abstract}
We analyse the influence of Brownian motion and thermophoresis on a nonlinearly permeable stretching sheet in a nanofluid. The governing partial differential equations are reduced into a system of ordinary differential equations using similarity transformation and then solved numerically using the Runge-Kutta with shooting technique. Effects of Brownian motion and thermophoresis on the flow, concentration, temperature, and mass transfer and heat transfer characteristics are investigated. The local Nusselt number and the local Sherwood numbers are presented and compared with existing results and are found to be in good agreement.
\end{abstract}

\section{Keywords}

Heat Transfer, Nanofluid, Boundary Layer, Flow, Stretching Sheet

\section{Introduction}

Nanofluids attract a great deal of interests with their enormous potential to provide enhanced performance properties, particularly with respect to heat transfer. Nanofluids are used for cooling of microchips in computers and other electronics which use microfluidic applications. Using nanofluids as coolants would allow for the radiators with smaller sizes and better positioning. Das et al. [1] experimentally showed a two- to four-fold increase in thermal conductivity enhancement for water-based nanofluids containing $\mathrm{Al}_{2} \mathrm{O}_{3}$ or $\mathrm{CuO}$ nanoparticles over a small temperature range of $21^{\circ} \mathrm{C}-51^{\circ} \mathrm{C}$. A comprehensive survey of convective transport in nanofluids has been made by Buongiorno [2], who gave a satisfactory explanation for the abnormal increase of the thermal conduc- 
tivity. Buongiorno and $\mathrm{Hu}$ [3] studied on the nanofluid coolants in advanced nuclear systems. Ahmad and Pop [4] investigated mixed convection boundary layer flow from a vertical flat plate embedded in a porous medium filled with nanofluids. Boundary layer flow of nanofluids over a moving surface in a flowing fluid was examined by Bachok et al. [5]. Khan and Pop [6] discussed the boundary-layer flow of a nanofluid past a stretching sheet. Makinde and Aziz [7] explained the boundary layer flow of a nanofluid past a stretching sheet with a convective boundary condition. Because of the materials with nanometer sizes having unique physical and chemical properties, they can flow through very thinly channels without clotting them. Khanafer et al. [8] explained the behavior of the liquid molecules in thinly channels. The viscosity and thermal conductivity on hydromagnetic flow over a nonlinear stretching sheet was discussed by Prasad et al. [9]. Kumari and Nath [10] analysed the flow and heat transfer of a MHD Newtonian fluid over impulsively stretched plane surface by using an analytical method namely homotopy analysis. The thermal conductivity of solid particles which is several times more than that of the base or convectional fluids was discussed by Das et al. [11] in the book nanofluids science and technology. In this book they clearly explained the thermal properties and behavior of the particles at different temperatures. The researchers Kumaran and Ramanaiah [12], Elbashbeshy [13] proved that stretching is not necessarily being a linear and they extended their research on flow over a quadratic stretching sheet and nonlinearly stretching sheet respectively. The heat transfer characteristics of the base fluids facing major obstacle to the effectives in heat exchange were discussed by Daungthongsuk and Wongwises [14]. Oztop and Abu-Nada [15] discussed the heat transfer characteristics on nanofluids by immersing the high conductivity nanomaterials in base fluids and they concluded that the effective thermal conductivity of the fluid increases appreciably and consequently enhances the heat transfer characteristics by suspending the high thermal conductivity of nanomaterials into the base fluids. The two-dimensional mixed convection boundary layer MHD stagnation point flow in porous medium bounded by a stretching vertical plate was studied by Hayat et al. [16]. They assumed that the stretching velocity and the surface temperature vary linearly along with the distance from stagnation point. The flow of an electrically conducting fluid in presence of uniform magnetic field over a stretching elastic sheet was studied by Pavlov [17]. By employing Darcy model for the porous medium, Cheng and Minkowycz [18] discussed a problem of natural convection past a vertical plate. They found the effect of Brownian motion and thermophoresis parameters on the velocity and temperature profiles. The transition effect of the boundary layer flow due to suddenly imposed magnetic field on the viscous flow past a stretching sheet and sudden withdrawal of a magnetic field on the viscous flow past a stretching sheet under a magnetic field is discussed by Kumaran et al. [19]. They found that in both cases the sheet stretches linearly along the direction of the fluid flow. Sandeep et al. [20] discussed radiation effects on unsteady natural convective flow of a nanofluid past an infinite vertical plate. Mohan Krishna et al. [21] extended this work by considering heat source effect and different nanofluids.

However, to the best of authors' knowledge, no attempt has been made to analyse the simultaneous effects of thermal radiation, Brownian motion and thermophoresis on the rate of heat and mass transfer flow of nanofluids over a non-linear stretching sheet. Hence, it is the reason why this problem is investigated.

\section{Mathematical Formulation}

Here, consideration is given to a steady, laminar, and incompressible and two dimensional boundary layer flow and heat transfer of a nanofluid past a permeable stretching/shrinking sheet. The pressure gradient and other external forces are neglected. Applying the boundary layer approximation, the governing equations for the conservation of mass, momentum, thermal energy and nanoparticle concentration are expressed as follows:

$$
\begin{gathered}
\frac{\partial u}{\partial x}+\frac{\partial v}{\partial y}=0 \\
u \frac{\partial u}{\partial x}+v \frac{\partial u}{\partial y}=v \frac{\partial^{2} u}{\partial y^{2}} \\
u \frac{\partial T}{\partial x}+v \frac{\partial T}{\partial y}=\alpha \frac{\partial^{2} T}{\partial y^{2}}+\tau\left\{D_{B} \frac{\partial C}{\partial x}\left(\frac{\partial T}{\partial y}\right)+\frac{D_{T}}{T_{\infty}}\left(\frac{\partial T}{\partial y}\right)^{2}\right\}+\frac{v}{c_{p}}\left(\frac{\partial u}{\partial y}\right)^{2}-\frac{1}{c_{p}} \frac{\partial q_{r}}{\partial y}
\end{gathered}
$$




$$
u \frac{\partial C}{\partial x}+v \frac{\partial C}{\partial y}=D_{B}\left(\frac{\partial^{2} C}{\partial y^{2}}\right)+\frac{D_{T}}{T_{\infty}}\left(\frac{\partial^{2} T}{\partial y^{2}}\right)
$$

The boundary conditions for Equations (1)-(4) are:

$$
\begin{aligned}
& u=\lambda U_{w}, v=V_{w}, T=T_{w}, C=C_{\infty} \text { at } y=0, \\
& U \rightarrow 0, T \rightarrow T_{\infty}, C \rightarrow C_{\infty}, \text { as } y \rightarrow \infty
\end{aligned}
$$

where $\lambda$ is the stretching/shrinking parameter, with $\lambda>0$ for a stretching surface and $\lambda<0$ for a shrinking surface.

By using Roseland approximation, the radiation heat flux $q_{r}$ is given by:

$$
q_{r}=-\frac{4 \sigma^{*}}{3 k^{*}} \frac{\partial T^{4}}{\partial y}
$$

where $\sigma^{*}$ and $k^{*}$ are the Stefan-Boltzmann constant and the mean absorption coefficient respectively. Considering the temperature differences within the flow sufficiently small such that $T^{4}$ may be expressed as the linear function of temperature. Then expanding $T^{4}$ in Taylor series about $T_{\infty}$ and neglecting higher-order terms takes the form:

$$
T^{4} \cong 4 T_{\infty}^{3} T-3 T_{\infty}^{4}
$$

In view of Equation (3) reduces to

$$
u \frac{\partial T}{\partial x}+v \frac{\partial T}{\partial y}=\alpha \frac{\partial^{2} T}{\partial y^{2}}+\tau\left\{D_{B} \frac{\partial C}{\partial x}\left(\frac{\partial T}{\partial y}\right)+\frac{\partial u}{\partial y}\left(\frac{\partial T}{\partial y}\right)^{2}\right\}+\frac{v}{c_{p}}\left(\frac{\partial u}{\partial y}\right)^{2}-\frac{1}{\rho c_{p}} \frac{16 T_{\infty}^{3} \sigma^{*}}{3 k^{*}} \frac{\partial^{2} T}{\partial y^{2}}
$$

Further, we seek for a similarity solution of Equations (1) to (4) subject to the boundary conditions (5). The governing partial differential forms can be solved by converting them to ordinary differential equations; this is done by using similarity functions:

$$
\begin{aligned}
& \eta=x^{\frac{n-1}{2}} y \sqrt{\frac{a(n+1)}{2 v}}, \quad u=a x^{n} f^{\prime}(\eta), U=a x^{n} f^{\prime}(\eta) \\
& v=-\sqrt{\frac{a v(n+1)}{2} x^{\frac{n-1}{2}}}\left(f(\eta)+\left(\frac{n-1}{n+1}\right) \eta f^{\prime}(\eta)\right), \quad \theta(\eta)=\frac{T-T_{\infty}}{T_{w}-T_{\infty}} \\
& \phi(\eta)=\frac{C-C_{\infty}}{C_{w}-C_{\infty}}, \quad R=\frac{16 T_{\infty}^{3} \sigma^{*}}{3 k k^{*}}
\end{aligned}
$$

where prime denotes differentiation with respect to eta $(\eta)$. To have similarity solution of Equations (1) to (5), we assume: $v=-\sqrt{\frac{a v(n+1)}{2}} x^{\frac{n-1}{2}} S$, where the constant parameter $S$ corresponds to suction $(S>0)$. By applying these similarity variables on the governing partial differential equations, transformed conservation equations and boundary conditions are then obtained as follows:

$$
\begin{aligned}
& f^{\prime \prime \prime}+f f^{\prime \prime}-\frac{2 n\left(f^{\prime}\right)^{2}}{n+1}=0 \\
& \theta^{\prime \prime}=-\operatorname{Pr}\left(N_{b} \theta^{\prime} \phi^{\prime}+N_{t}\left(\theta^{\prime}\right)^{2}+f \theta^{\prime}+E c\left(f^{\prime \prime}\right)^{2}\right) /(1+\operatorname{Pr} R) \\
& \phi^{\prime \prime}+\frac{N_{t}}{N_{b}} \theta^{\prime \prime}+\text { Lef } \phi^{\prime}=0
\end{aligned}
$$

\section{Boundary Conditions}

$$
\text { At } y=0, \eta=0, f^{\prime}(0)=\lambda
$$




$$
\begin{aligned}
& v=-\sqrt{\frac{a v(n+1)}{2} x^{\frac{n-1}{2}}}\left(f(\eta)+\left(\frac{n-1}{n+1}\right) \eta f^{\prime}(\eta)\right) \\
& V_{w}=-\sqrt{\frac{a v(n+1)}{2}} x^{\frac{n-1}{2}} S \\
& f(0)=S, \theta(0)=1, \phi(0)=1, \text { as } \eta \rightarrow 0 \\
& f^{\prime}(\eta)=0, \theta(\eta)=0, \phi(\eta)=0 \text { as } \eta \rightarrow \infty
\end{aligned}
$$

However, the quantities of physical and engineering interest are the reduced Nusselt number $\left(-\theta^{\prime}\right)$ and reduced Sherwood number $\left(-\phi^{\prime}\right)$. From the knowledge of the Nusselt number, the local convection coefficient may be found and the local heat flux may then be computed. The reduced Sherwood number on the other hand is the parameter that defines the dimensionless concentration gradient at the surface, and it provides a measure of the convection mass transfer occurring at the surface. The skin friction coefficient can be used to compute stresses developing at the wall.

$$
\begin{aligned}
& C_{f}=\frac{\tau_{w}}{\rho\left(U_{w}\right)^{2}}=\frac{\mu \frac{\partial u}{\partial y}}{\rho\left(U_{w}\right)^{2}}=R e_{x}^{-1 / 2} \sqrt{\frac{(n+1)}{2}} f^{\prime \prime}(0) \\
& C_{f}=R e_{x}^{-1 / 2} \sqrt{\frac{(n+1)}{2}} f^{\prime \prime}(0)
\end{aligned}
$$

The local heat transfer rate (Local Nusselt) number is given by

$$
\begin{aligned}
& N u_{x}=\frac{x q_{w}}{k\left(T_{w}-T_{\infty}\right)}=-\left(\frac{R e_{x} v}{a}\right)^{1 / 2} \sqrt{\frac{a(n+1)}{2 v}} \theta^{\prime}(0) \\
& N u_{x} R e_{x}^{-1 / 2}=-\sqrt{\frac{(n+1)}{2}} \theta^{\prime}(0)
\end{aligned}
$$

And then the local Sherwood number is;

$$
\begin{aligned}
& S h_{x}=\frac{x q_{m}}{D_{B}\left(C_{w}-C_{\infty}\right)}=-\left(\frac{R e_{x} v}{a}\right)^{1 / 2} \sqrt{\frac{a(n+1)}{2 v}} \phi^{\prime}(0) \\
& S h_{x} R e_{x}^{-1 / 2}=-\sqrt{\frac{(n+1)}{2}} \phi^{\prime}(0)
\end{aligned}
$$

where $q_{w}$ and $q_{m}$ denotes the wall heat and mass flux rates respectively.

\section{Reduction of the Ordinary Differential Equations}

The set of Equations (10) to (12) under the boundary conditions (16) have been solved numerically using shooting technique. We consider: $y_{1}=f, y_{2}=f^{\prime}, y_{3}=f^{\prime \prime} y_{4}=\theta, y_{5}=\theta^{\prime}, y_{6}=\phi, y_{7}=\phi^{\prime}$. Equations (10) to (12) are transformed into systems of first order differential equations. We assume the unspecified initial guesses for the transformed boundary conditions and integrated the equations numerically as an initial valued problem.

\section{Results and Discussion}

The results obtained shows the influences of the non-dimensional governing parameters, namely Radiation parameter $R$, Suction parameter $S$, Lewis number $L e$, thermophoresis parameter $N_{t}$ and Brownian motion parameter $N_{b}$ on temperature profile, nanoparticle concentration profile, a the local Nusselt number and the Sherwood number. For numerical results we used $L e=2, E c=0.5, n=2, S=2$ and $\lambda=2$ for different values of $N_{b}, N_{t}$ and 
$R$ in entire study. These values are kept constant except the varied values shown in the figures. The numerical results obtained, i.e., the present results for Nusselt number and Sherwood number were compared with those obtained by Khairy, Anuar Ishak and Ioan Pop [22] for the case of a stretching surface by setting $R=0, L e=2$, $n=2, N_{b}=0.5, N_{t}=0.5$ and $\operatorname{Pr}=6.2$.

In order to get a clear insight of the physical problem, numerical computation have been carried out as described above for various values of different parameters (Table 1 ).

To assess the accuracy of the method, the results are compared with those reported in literature by Khairy, Anuar and Ioan Pop [22]. The results are found to be in good agreement.

Figure 1 and Figure 2 illustrate the effect of themophoresis on the temperature and the rate of heat transfer respectively. Both these figures show the variation of the temperature and the heat transfer profiles with increasing thermophoresis parameter.

With increase thermophoresis parameter, both figures show that the boundary layer thickness increases, leading to increase in temperature. The rate of heat transfer increases rapidly initially (up to the point $\eta=0.4$ ) and later decreases to a non-zero value. In Figure 3 and Figure 4, with increasing thermophoresis, the concentration boundary layer thickness increases while for the rate of mass transfer, the boundary layer thickness reduces. These show that while concentration increases within the boundary layer, the rate of mass transfer reduces.

Table 1. Values of $-\theta^{\prime}(0)$ and $-\phi^{\prime}(0)$ for different values of $\mathrm{S}$ and $\lambda$ when $n=2, L e=2, N_{t}=0.5, N_{b}=0.5$ and $P r=6.2$ with Khairyzaimi, Anuar and Ioan Pop [22].

\begin{tabular}{cccccc}
\hline & \multicolumn{2}{c}{ Khairyzaimi, Anuar and Ioan Pop [22] } & \multicolumn{2}{c}{ Present results } \\
\hline $\mathrm{S}$ & $\lambda$ & $-\theta^{\prime}(0)$ & $-\phi^{\prime}(0)$ & $-\theta^{\prime}(0)$ & $-\phi^{\prime}(0)$ \\
2.5 & -0.5 & 7.887191 & -6.070289 & 7.88719 & -6.07029 \\
3.0 & 2.0 & 7.984141 & -4.499616 & 7.9841 & -4.4996 \\
3.5 & -0.5 & 10.790697 & -8.043106 & 10.7907 & -8.0431 \\
4.0 & 2.0 & 10.750182 & -6.361137 & 10.7502 & -6.3611 \\
5.0 & -0.5 & 15.266129 & -11.207552 & 15.2661 & -11.2076 \\
2.5 & 2.0 & 7.151258 & -4.446270 & 7.1513 & -4.4463 \\
3.0 & -0.5 & 9.681430 & -6.311584 & 9.6814 & -6.3116 \\
3.5 & 2.0 & 9.366247 & -5.434047 & 9.3663 & -5.4305 \\
4.0 & -0.5 & 12.274461 & -9.083765 & 12.2745 & -9.0838 \\
5.0 & 2.0 & 14.788043 & -10.216636 & 14.7795 & -10.2166 \\
\hline
\end{tabular}

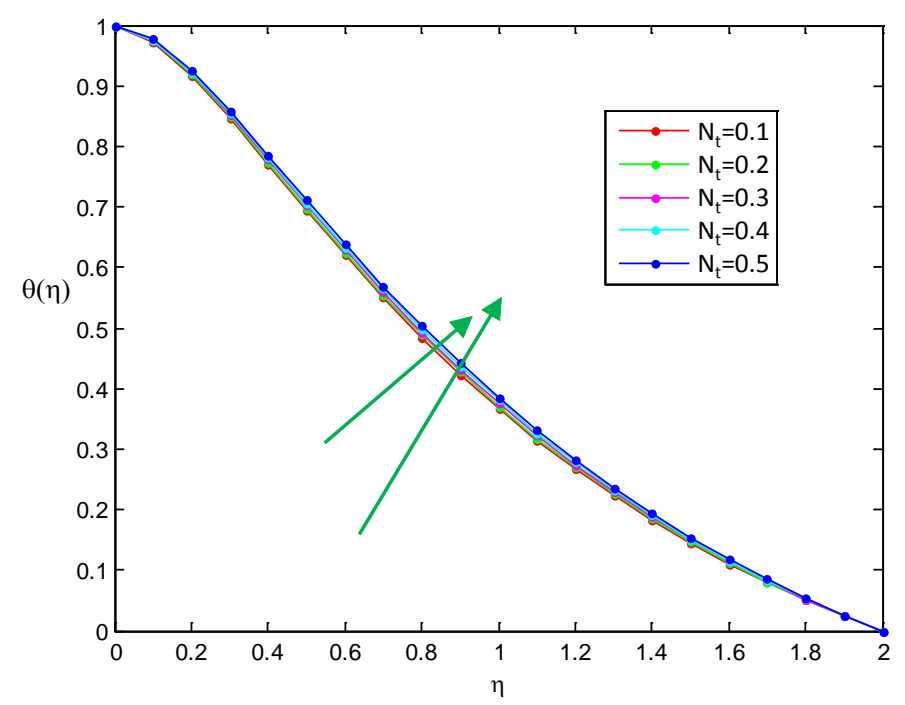

Figure 1. Effect of thermophoresis on temperature profiles $\theta(\eta)$. 


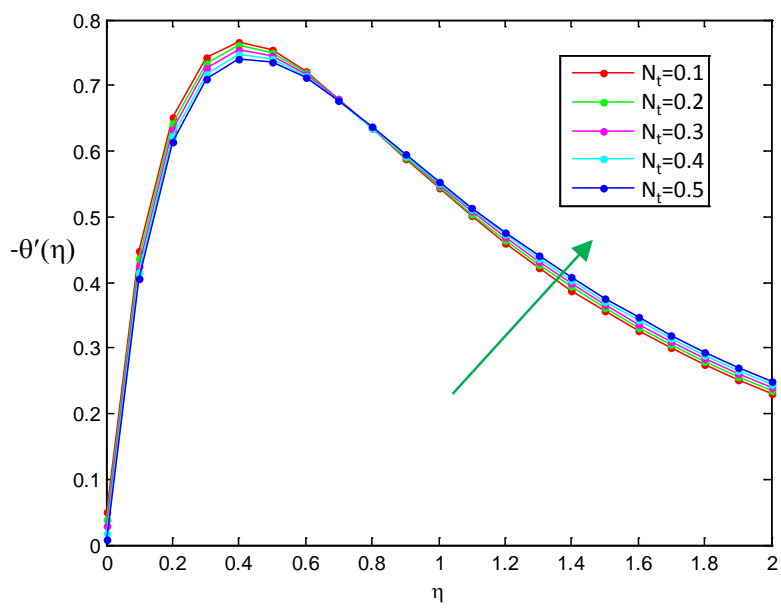

Figure 2. Effect of thermophoresis on rate of heat transfer $-\theta^{\prime}(\eta)$.

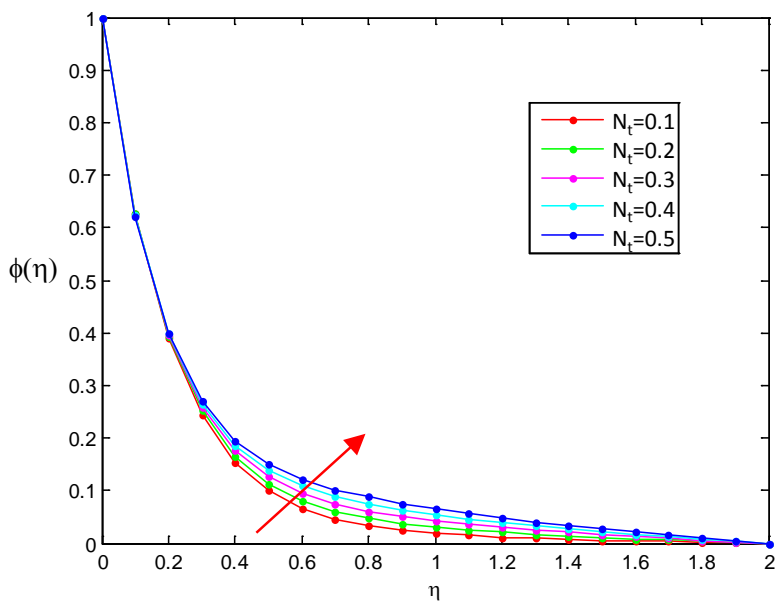

Figure 3. Effect of thermophoresis on concentration profiles $\phi(\eta)$.

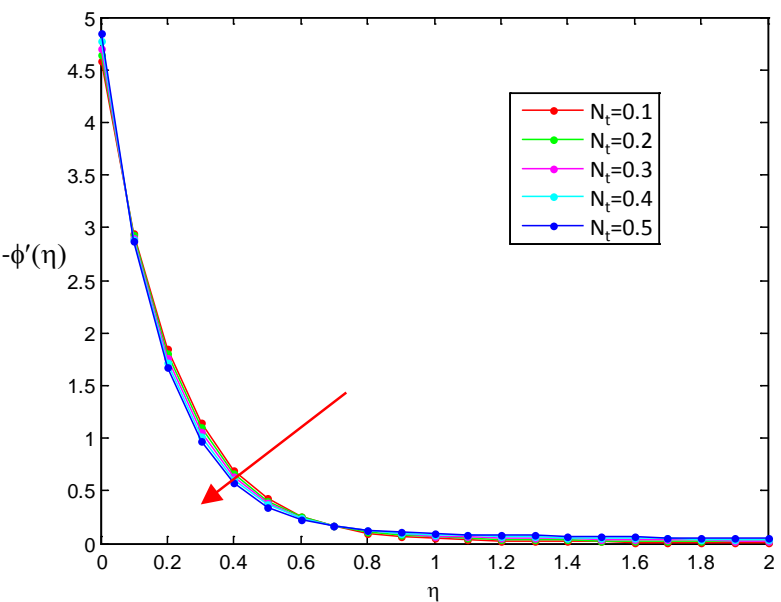

Figure 4. Effect of thermophoresis on rate of mass transfer $-\phi^{\prime}(\eta)$. 
We now concentrate on the effects of Brownian motion on the temperature, concentration, rate of heat transfer and the rate of mass transfer. In Figure 5 and Figure 6, with increasing Brownian motion parameter the temperature and rate of heat transfer boundary layer thickness increase. This shows increasing temperature leading to heating while the heat transfer rate increases rapidly (up to the point, $\eta=0.3$ ) and decreases thereafter. With Figure 7 and Figure 8, we see decreasing concentration with increasing Brownian motion parameter while the rate of mass transfer increases with increasing Brownian motion parameter.

Next, we look at the effects of the stretching parameter. Figure 9 and Figure 10 depict the effects of the stretching parameter $(\lambda)$ on the velocity and the skin friction. It is seen that increasing stretching parameter leads to increase in velocity and decrease in the skin friction. On the other hand, Figure 11 and Figure 12 show increase in temperature and decrease in concentration with increasing stretching parameter.

Figure 13 and Figure 14 show that with increasing stretching parameter, the rate of heat transfer increases initially and later decreases rapidly while the rate of mass transfer increases with increasing stretching parameter.

\section{Conclusions}

This study analysed the influence of Brownian motion and thermophoresis in nonlinearly permeable stretching

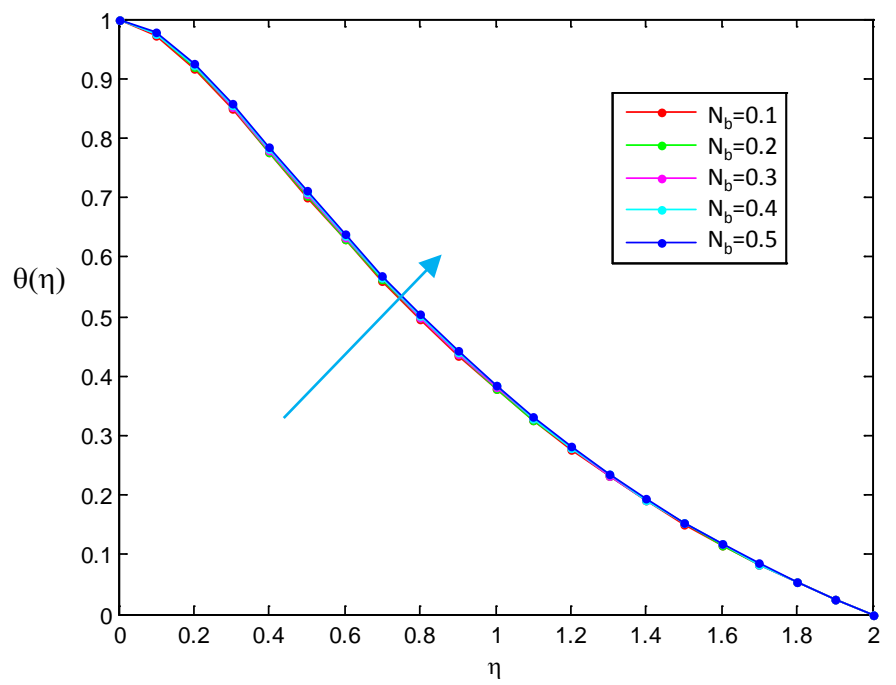

Figure 5. Effect of Brownian motion on temperature profiles $\theta(\eta)$.

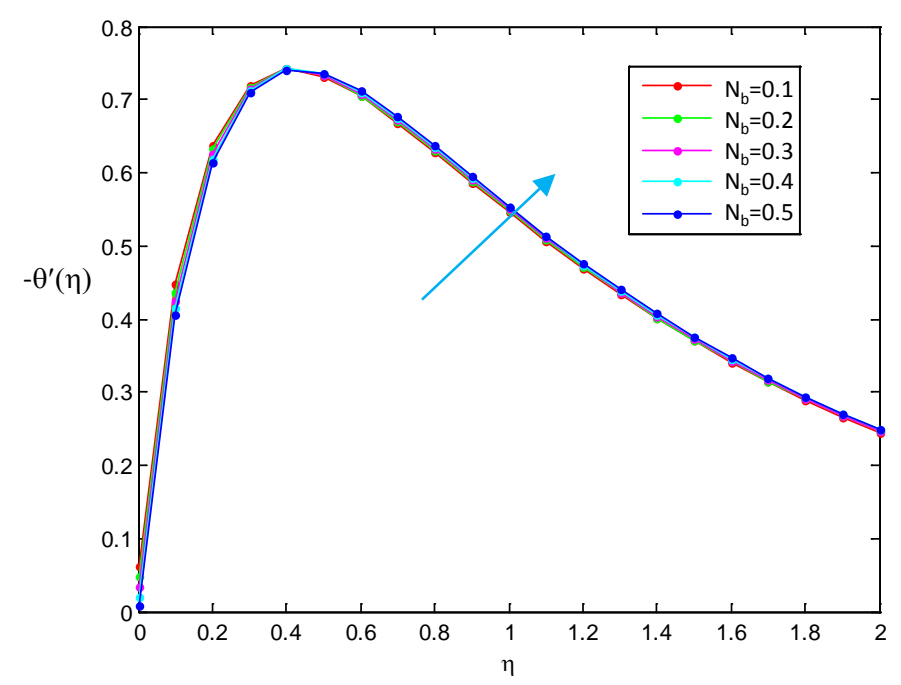

Figure 6. Effect of Brownian motion on rate of heat transfer $-\theta^{\prime}(\eta)$. 


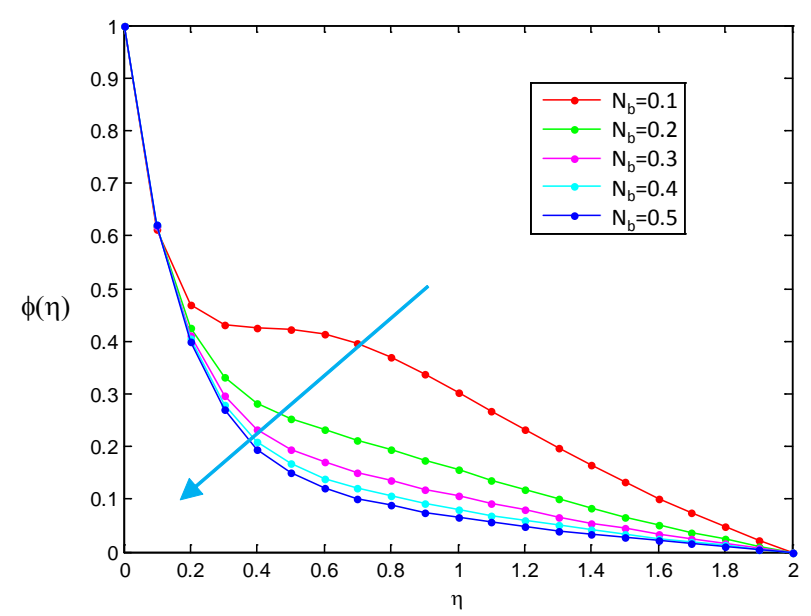

Figure 7. Effect of Brownian motion on concentration profiles $\phi(\eta)$.

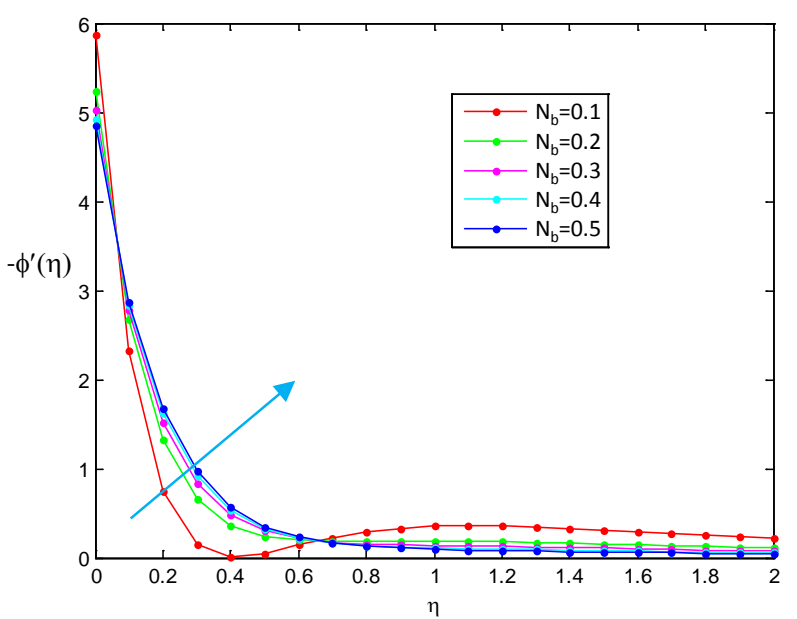

Figure 8. Effect of Brownian motion on rate of mass transfer $-\phi^{\prime}(\eta)$.

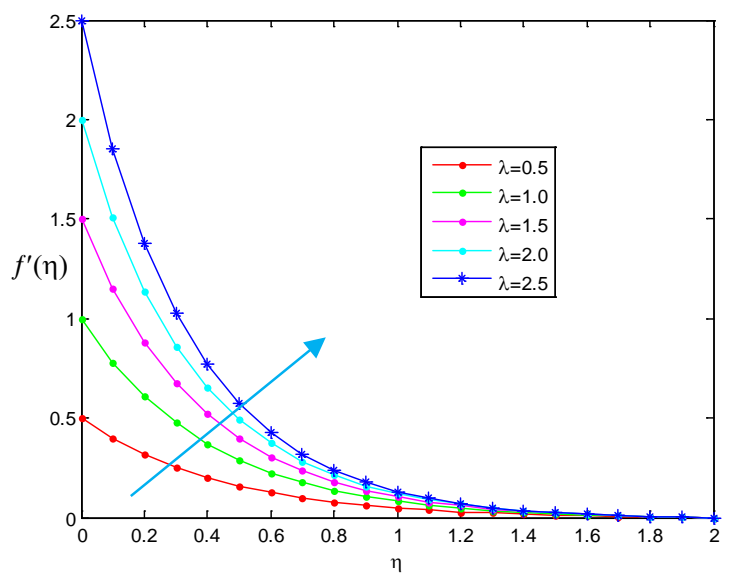

Figure 9. Velocity profiles $f^{\prime}(\eta)$ with variation in stretching parameter $(\lambda)$ for a stretching case, when $N_{b}=0.5$ and $N_{t}=$ 0.5 . 


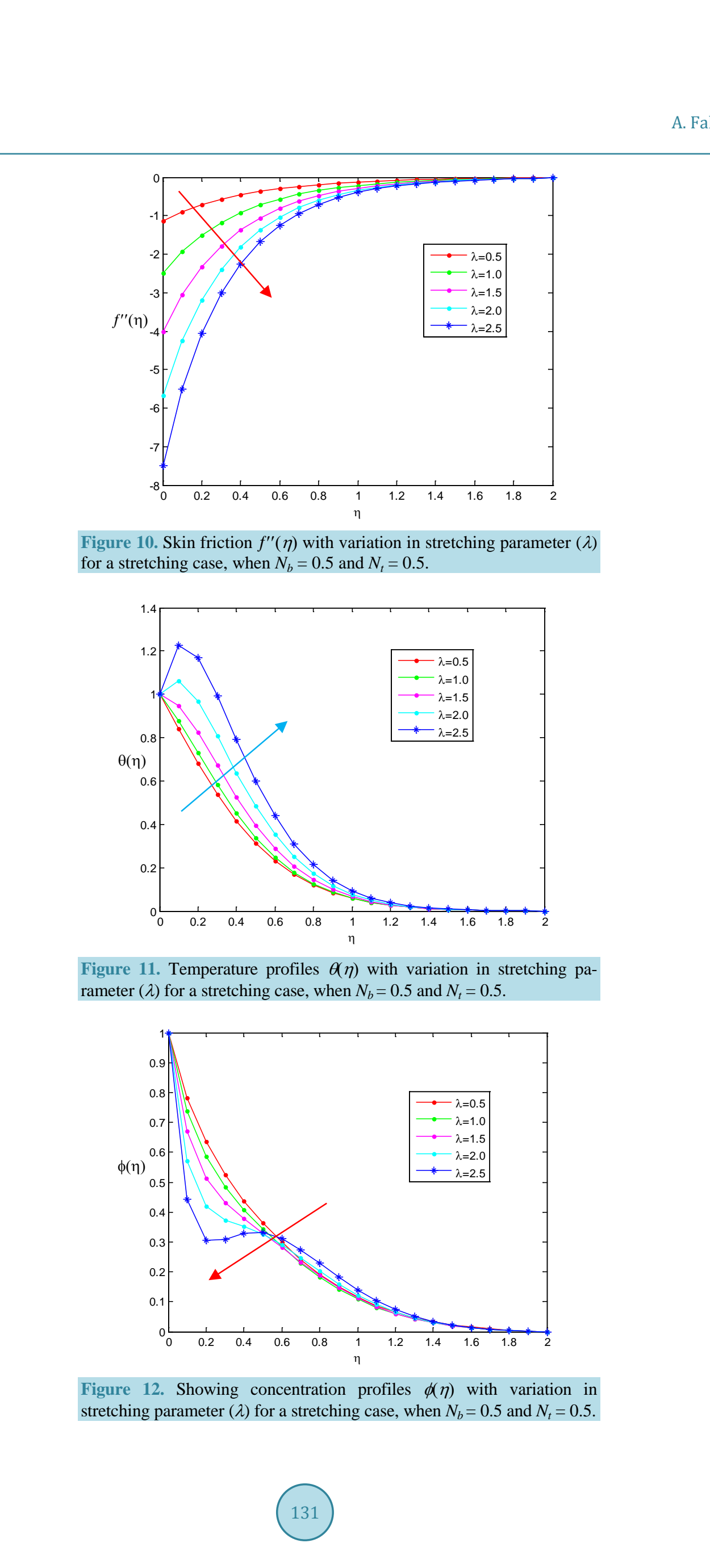




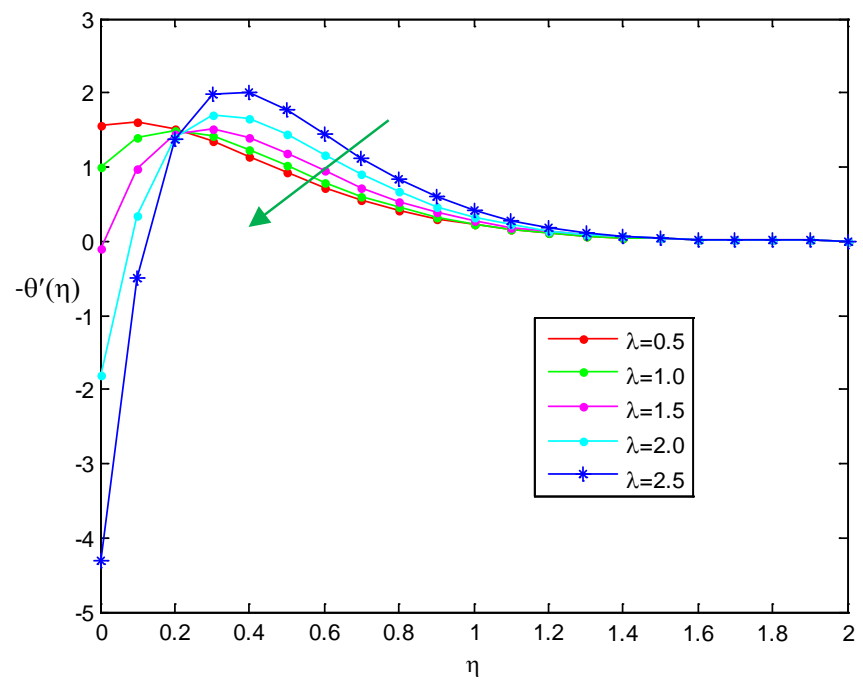

Figure 13. Rate of heat transfer (reduced Nusselt) $-\theta^{\prime}(\eta)$ with variation in stretching parameter $(\lambda)$ for a stretching case, when $N_{b}=0.5$ and $N_{t}=0.5$.

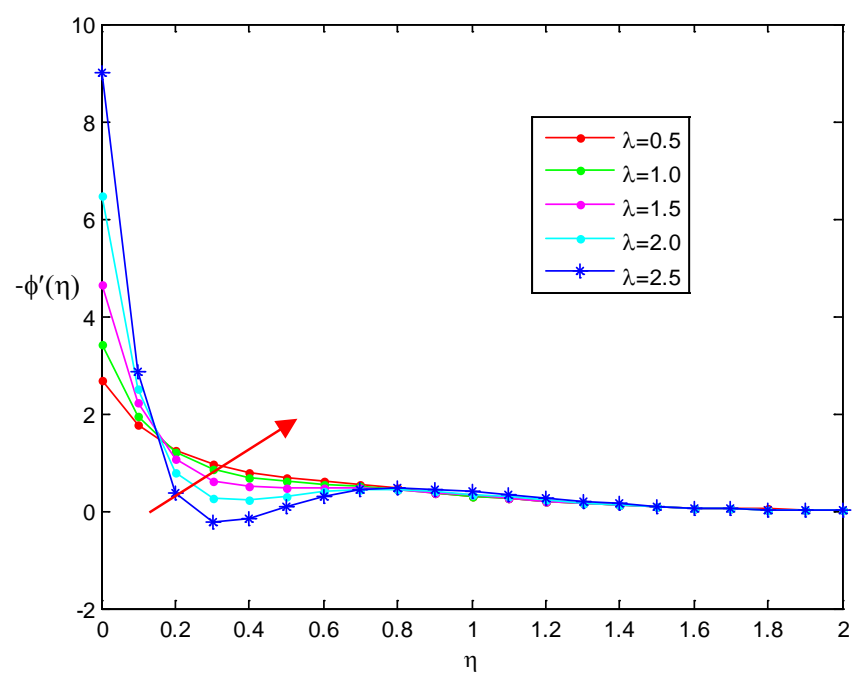

Figure 14. Rate of mass transfer (Reduced Sherwood) $-\phi^{\prime}(\eta)$ with variation in stretching parameter $(\lambda)$ for a stretching case, when $N_{b}=0.5$ and $N_{t}=0.5$.

sheet in a nanofluid. The non-linear partial differential equations and their associated boundary conditions have been transformed to non-dimensional ordinary differential equations using the similarity transformations and the resultant initial value problem is solved by an iterative Runge-Kutta method along with shooting technique. The present results are compared with the existing results in literature and were found to agree well. The influences of the governing parameters on the temperature, concentration, heat and mass transfer rates have been systematically examined. From the present numerical investigation, the following conclusion can be made:

1) There is a rise in the temperature with an increase in the thermophoresis parameter or Brownian motion parameter or stretching parameter.

2) Species concentration decreases with an increase in Brownian motion while the concentration increases for an increase in the values of the thermophoresis parameter.

3) A rising value in $N_{b}$ and the decreasing in $N_{t}$ produce a decrease in the nanoparticle concentration, and as a result increase in the Sherwood number. 


\section{References}

[1] Das, S.K., Putra, N., Thiesen, P. and Roetzel, W. (2003) Temperature Dependence of Thermal Conductivity Enhancement for Nanofluids. Journal of Heat Transfer, 125, 567-574. http://dx.doi.org/10.1115/1.1571080

[2] Buongiorno, J. (2006) Convective Transport in Nanofluids. Journal of Heat Transfer, 128, 240-250. http://dx.doi.org/10.1115/1.2150834

[3] Buongiorno, J. and Hu, W. (2005) Nanofluid Coolants for Advanced Nuclear Power Plants. Proceedings of ICAPP’05, Seoul, 15-19 May 2005, 15-19.

[4] Ahmad, S. and Pop, I. (2010) Mixed Convection Boundary Layer Flow from a Vertical Flat Plate Embedded in a Porous Medium Filled with Nanofluids. International Communications in Heat and Mass Transfer, 37, 987-991. http://dx.doi.org/10.1016/j.icheatmasstransfer.2010.06.004

[5] Bachok, N., Ishak, A. and Pop, I. (2010) Boundary-Layer Flow of Nanofluids over a Moving Surface in a Flowing Fluid. International Journal of Thermal Sciences, 49, 1663-1668. http://dx.doi.org/10.1016/j.ijthermalsci.2010.01.026

[6] Khan, W.A. and Pop, I. (2010) Boundary-Layer Flow of a Nanofluid past a Stretching Sheet. International Journal of Heat and Mass Transfer, 53, 2477-2483. http://dx.doi.org/10.1016/j.ijheatmasstransfer.2010.01.032

[7] Makinde, O.D. and Aziz, A. (2011) Boundary Layer Flow of a Nanofluid past a Stretching Sheet with a Convective Boundary Condition. International Journal of Thermal Sciences, 50, 1326-1332. http://dx.doi.org/10.1016/j.ijthermalsci.2011.02.019

[8] Khanafer, K., Vafai, K. and Lightstone, M. (2003) Buoyancy Driven Heat Transfer Enhancement in a Two Dimensional Enclosure Utilizing Nanofluids. International Journal of Heat and Mass Transfer, 46, 3639-3653. http://dx.doi.org/10.1016/S0017-9310(03)00156-X

[9] Prasad, K.V., Vajravelu, K. and Datti, P.S. (2010) The Effects of Variable Fluid Properties on the Hydro-Magnetic Flow and Heat Transfer over a Non-Linearly Stretching Sheet. International Journal of Thermal Sciences, 49, 603-610. http://dx.doi.org/10.1016/j.ijthermalsci.2009.08.005

[10] Kumari, M. and Nath, G. (2009) Analytical Solution of Unsteady Three-Dimensional MHD Boundary Layer Flow and Heat Transfer Due to Impulsively Stretched Plane Surface. Communications in Nonlinear Science and Numerical Simulation, 14, 3339-3350. http://dx.doi.org/10.1016/j.cnsns.2008.11.011

[11] Das, S.K., Choi, S.U.S., Yu, W. and Pradeep, T. (2007) Nanofluids: Science and Technology. Willey, Hoboken. http://dx.doi.org/10.1002/9780470180693

[12] Kumaran, V. and Ramanaih, G. (1996) A Note on the Flow over Stretching Sheet. Archives of Mechanics, 116, 229233. http://dx.doi.org/10.1007/bf01171433

[13] Elbashbeshy, E.M.A. (2001) Heat Transfer over an Exponentially Stretching Continuous Surface with Suction. Archives of Mechanics, 53, 641-651.

[14] Daungthongsuk, W. and Wongwises, S. (2007) A Critical Review of Convective Heat Transfer Nanofluids. Renewable \& Sustainable Energy Reviews, 11, 797-817. http://dx.doi.org/10.1016/j.rser.2005.06.005

[15] Oztop, H.F. and Abu-Nada, E. (2008) Numerical Study of Natural Convection in Partially Heated Rectangular Enclosures Filled with Nanofluids. International Journal of Heat and Fluid Flow, 29, 1326-1336. http://dx.doi.org/10.1016/j.ijheatfluidflow.2008.04.009

[16] Hayat, T., Abbas, Z., Pop, I. and Asghar, S. (2010) Effects of Radiation and Magnetic Field on the Mixed Convection Stagnation Point Flow over a Vertical Stretching Sheet in a Porous Medium. International Journal of Heat and Mass Transfer, 53, 466-474. http://dx.doi.org/10.1016/j.ijheatmasstransfer.2009.09.010

[17] Palov, K.B. (1974) Magnetohydrodynamic Flow of an Incompressible Viscous Fluid Caused by Deformation of a Plane Surface. Magnitnaya Gidrodinamika (USSR), 4, 146-147.

[18] Cheng, P. and Minkowycz, W.J. (1977) Free Convection about a Vertical Flat Plate Embedded in a Porous Medium with Application to Heat Transfer from Adike. Journal of Geophysical Research, 82, 2040-2044. http://dx.doi.org/10.1029/JB082i014p02040

[19] Kumaran, V., Kumar, A.V. and Pop, I. (2010) Transmission of MHD Boundary Layer Flow past a Stretching Sheet. Communications in Nonlinear Science and Numerical Simulation, 15, 300-311. http://dx.doi.org/10.1016/j.cnsns.2009.03.027

[20] Sandeep, N., Sugunamma, V. and Mohankrishna, P. (2013) Effects of Radiation on an Unsteady Natural Convective Flow of a EG-Nimonic 80a Nanofluid past an Infinite Vertical Plate. Advances in Physics Theories and Applications, 23, 36-43.

[21] Mohankrishna, P., Sugunamma, V. and Sandeep, N. (2014) Radiation and Magneticfield Effects on Unsteady Natural Convection Flow of a Nanofluid past an Infinite Vertical Plate with Heat Source. Chemical and Process Engineering Research, 25, 39-52. 
[22] Zaimi, K., Ishak, A. and Pop, I. (2014) Boundary Layer Flow and Heat Transfer over a Nonlinearly Permeable Stretching/Shrinking Sheet in a Nanofluid. Scientific Reports, 4, 4404.

\section{Nomenclature}

a: a positive constant

$u, v$ : velocity components in $x$ and $y$ directions respectively

$\lambda$ : the stretching/shrinking parameter ( $\lambda>0$ for stretching surface and $\lambda<0$ ) for shrinking surface

$D_{B}$ : Brownian diffusion coefficient

$D_{T}$ : thermophoretic diffusion coefficient

$N_{b}$ : Brownian motion parameter, defined Nomenclature

Le: Lewis number

$N_{t}$ : thermophoresis parameter

$\mathrm{Nu}_{\mathrm{x}}$ : reduced Nusselt number

Pr: Prandtl number

$m$ : wall mass flux

$w$ : wall heat flux

$R e_{x}$ : local Reynolds number

$S h_{x}:$ reduced Sherwood number

$T_{w}$ : sheet surface (wall) temperature

$T_{\infty}:$ ambient temperature

$x$ : coordinate along the sheet

$y$ : coordinate normal to the sheet

$C$ : nanoparticle volume fraction

$C_{w}$ : nanoparticle volume fraction at the sheet surface (wall)

$C_{\infty}$ : nanoparticle volume fraction at large values of $y$ (ambient)

Greek symbols

$\tau=(\rho c)_{p} /(\rho c)_{f}$

$(\rho c)_{f}$ : heat capacity of the base fluid

$(\rho c)_{p}^{f}$ : heat capacity of the nanoparticle material

$u_{w}=a x$ 